\title{
Preparatory Efficacy Effects on Practice Effort and Performance
}

\author{
Jared M. Wood ${ }^{1}$ and Deborah L. Feltz ${ }^{2, *}$ \\ ${ }^{1}$ Sport Psychology Consultant, Clarkston, MI 48346 USA \\ ${ }^{2}$ Department of Kinesiology, Michigan State University, 308 W. Circle Drive, Intramural Rec Sports-Circle, East \\ Lansing, MI 48824 USA
}

\begin{abstract}
Bandura [1] has theorized that during preparation for sport competition some doubt about one's capabilities to perform effectively may be beneficial to spurring athletes to put forth a strong preparatory effort. We designed two experiments to test the idea that lower preparatory efficacy levels would benefit practice effort. Participants who competed in golf putting competitions were separated into preparation and competition phases. Participants categorized put balls to three targets of varying difficulty (i.e., high, medium, and low efficacy targets). Preparation phases consisted of 30 free choice practice putts. Practice effort was measured as the number of practice putts allocated to each target. In both studies, the high efficacy target resulted in the lowest practice effort in comparison to practice effort at the medium and low efficacy targets $(p<.001)$, and preparatory efficacy was associated with a significant linear increase in effort across the respective high, medium, and low efficacy targets.
\end{abstract}

Keywords: Self-efficacy, performance efficacy, preparation, motivation, preparatory efficacy.

\section{INTRODUCTION}

In sports, efficaciousness is believed to be essential for success. Coaches and culture teach athletes that confidence, swagger, and bravado are keys to playing great, and coaches strive to instill a can-do belief in their players. Sport psychology consultants are major proponents of building a strong sense of self-efficacy. Often defined as situationspecific self-confidence [1,2], self-efficacy has been shown to have a temporally recursive relationship with performance [3-9]. Self-efficacy further indirectly exerts its influence on performance through effort and persistence [10,11], choice of goals and activities [12, 13], decision-making [14], and interpretations of emotional reactions [15]. Self-efficacy is considered one of the most important contributors to sport performance [2], even when past performance is factored out of the causal model statistically [16]. Thus, the suggestion that athletes should have a high sense of self-efficacy prior to performance is supported by the extant research.

Despite its known performance benefits, high selfefficacy is not immune to criticism [17-20]. Overconfidence is a persistent and pervasive idea in sports. Favored teams who perform below their own standards and lose to underdog opponents are often described as being overconfident. Therefore, the term overconfidence, is used to convey the idea that high confidence is not always good for athletic performance. This begs the question, how and when can confidence become problematic for performance?

*Address correspondence to this author at the Department of Kinesiology, Michigan State University, 308 W. Circle Drive, Intramural Rec SportsCircle, East Lansing, MI 48824 USA; Tel: (517) 355-4732;

Fax: (517) 353-2944; E-mail: dfeltz@msu.edu
Weinberg and Gould [21] have suggested that overconfidence may exert its influence on performance indirectly through haphazard preparation. Bandura [1] theorized, similarly, on this issue when he wrote that high selfefficacy is best for competitive performance, but some sense of doubt may be necessary for engendering motivation to put forth great preparatory effort. After all, why would athletes prepare earnestly for a competition if winning is assured?

To prepare with optimal effort, Bandura [1] argued athletes must have some sense of doubt about the outcome of the impending competition. Thus, Bandura [1] believes doubt is detrimental to performance during sport competition [22], yet it may be beneficial to effortful practice during the preparation phase of competition. Because the preparation phase in all sports is longer than the performance phase, preparatory effort is an important building block of performance [23]. Through effortful practice behaviors, selfefficacy enhancing information, such as mastery experience, becomes available, and self-efficacy beliefs increase accordingly [23], restoring or building self-efficacy before competition [1].

Although many studies have concluded that high selfefficacy results in high effort or persistence in performance situations [10, 11, 24], Bandura and Cervone [25] have shown that some research participants slackened effort when performance approached a goal level, a condition Bandura and Cervone referred to as overcomplacency. Bandura and Cervone concluded, "Motivation is perhaps best maintained by a strong sense of self-efficacy to withstand failure, coupled with some uncertainty (construed in terms of the challenge of the task, rather than fundamental doubt about one's capabilities) to spur the effort needed to fulfill personal 
challenges. It remains a problem of future research to delineate the factors that contribute to overcomplacency," (p. 110). Although Bandura and Cervone were studying performance, overcomplacency also could be a phenomenon associated with haphazard preparation in the presence of overly inflated preparatory efficacy beliefs.

To begin studying preparatory efficacy, it is important to accurately define the concept and compare and contrast it to its more commonly studied self-efficacy counterpart, performance efficacy. Similar to performance efficacy, preparatory efficacy is defined as beliefs in one's capabilities in an upcoming performance [2]. However, unlike performance efficacy, which is measured as close as possible to the actual performance - in the research the measurements have typically taken place within $24 \mathrm{hr}$ of the performance upon which the self-efficacy beliefs are based - preparatory efficacy beliefs must be sampled within a defined preparation period for said performance [23]. Thus, the separation between preparation and performance is vital to the study of preparatory efficacy.

Preparation versus learning is also an important distinction in preparatory efficacy research. Bandura [1] has carefully distinguished between the two concepts in his writing. Preparation is the act of preparing for an upcoming event. Learning involves the acquisition of new skills or knowledge. Although preparation may involve learning, not all learning occurs in preparation for an event. To the point that learning and preparation are related but distinct concepts, several learning studies have shown that relatively lower self-efficacy levels can be beneficial to effort and performance on cognitive learning tasks $[26,27,28]$ and novel motor performance tasks [29].

Given the dearth of studies on preparatory efficacy, the two experiments in this article were designed to examine whether doubt can be beneficial to preparatory effort. To test this idea, a distinct separation of preparation and performance was created for a golf putting task that typically improves with repeated practice (i.e., stroking a putt with the proper speed, or pace, so it lands within or as close as possible to a target). Researchers created targets of varying difficulties that corresponded to high, medium, and low selfefficacy perceptions. In both experiments, the main hypothesis predicted that the lowest effort would occur at the high preparatory efficacy targets, moderate effort would occur at the medium preparatory efficacy targets, and the greatest effort would occur at the low preparatory efficacy targets.

\section{EXPERIMENT 1}

\section{Overview}

For the initial inquiry into preparatory efficacy, the researchers standardized preparatory efficacy levels across three putting targets of varying difficulty. The lengths from the starting point of the putt to the targets were allowed to vary according to participant self-efficacy levels. Participants rated preparatory efficacy levels according to how many putts each believed he could land within given target zones of varying distances. The targets were set according to the farthest distance for which each participant indicated he believed he could make five out of five putts, three out of five putts, and only one out of five putts. We hypothesized that the most practice effort (i.e., number of putts) would be directed at the low efficacy target, moderate effort would be directed at the medium efficacy target, and the least effort would be directed at the high efficacy target. Further, based on the idea that preparatory efficacy and overconfidence affect performance through lack of effort on preparatory tasks, we hypothesized that participants would underperform in comparison to expectations at the high efficacy target but not at either the medium or low efficacy targets.

\section{Method}

Participants. Prior to beginning the study, researchers obtained approval of the research protocol from the authorized Institutional Review Board (IRB). Via email and direct contact, researchers recruited male participants $(N=$ 24) from a golf training program and a recreational golf league in the Midwest. The study required 3 years of golf playing experience. Participant average age was 38.7 years $(S D=8.8)$ with ages ranging from 19 to 69 . Golf experience averaged 20.7 years $(S D=8.6)$. Highest level of official competitive experience data included 14 non-competitive participants, 8 league level competitors, and 2 high school level competitors.

Golf materials. The golf equipment used in this study included a bullseye style putter and 30 regulation golf balls. Bullseye style putters are flat on both sides, which allowed for the same putter to be used for both left and right-handed participants.

The putting surface was made of Astroturf style indooroutdoor carpeting. The surface was $24 \mathrm{ft}(7.32 \mathrm{~m})$ long and four $\mathrm{ft}(1.22 \mathrm{~m})$ wide. Twenty $\mathrm{ft}(6.10 \mathrm{~m})$ of the surface was marked into $1 \mathrm{ft}(0.30 \mathrm{~m})$ long zones. White painted stripes marked the beginning of each successive zone. Zones were labeled in white paint to help participants identify them by name. The first zone was labeled Zone 1, the second Zone 2, and so forth up to Zone 20 . Two $\mathrm{ft}(0.61 \mathrm{~m})$ at the front end of the surface served as a place for participants to stand, while $2 \mathrm{ft}(0.61 \mathrm{~m})$ at the end created depth past the last zone, which helped participants judge the distance putts rolled past the last zone. A small white circle 6 in. $(0.15 \mathrm{~m})$ in front of Zone 1 marked the starting point for each putt. The starting point was $1 \mathrm{ft}(0.30 \mathrm{~m})$ from the center of Zone $1,2 \mathrm{ft}$ from the center of Zone 2, and so forth. Therefore, the name of each zone indicated the number of feet from its center to the starting point. The putting surface is illustrated in Fig. (1).

Self-efficacy measures. Participants rated preparatory and performance self-efficacy strengths on 11-point scales (0-10) with the anchors Complete Uncertainty, Moderate Certainty, and Complete Certainty set at 0, 5, and 10 respectively. For each of the three targets, participants rated self-efficacy beliefs in capability to land at least one out of five, at least three out of five, and all five putts in the target zone. Preparatory and performance efficacy strength scores were obtained for each target by summing across the three items for each target and then dividing by three. The preparatory and performance efficacy measures were 


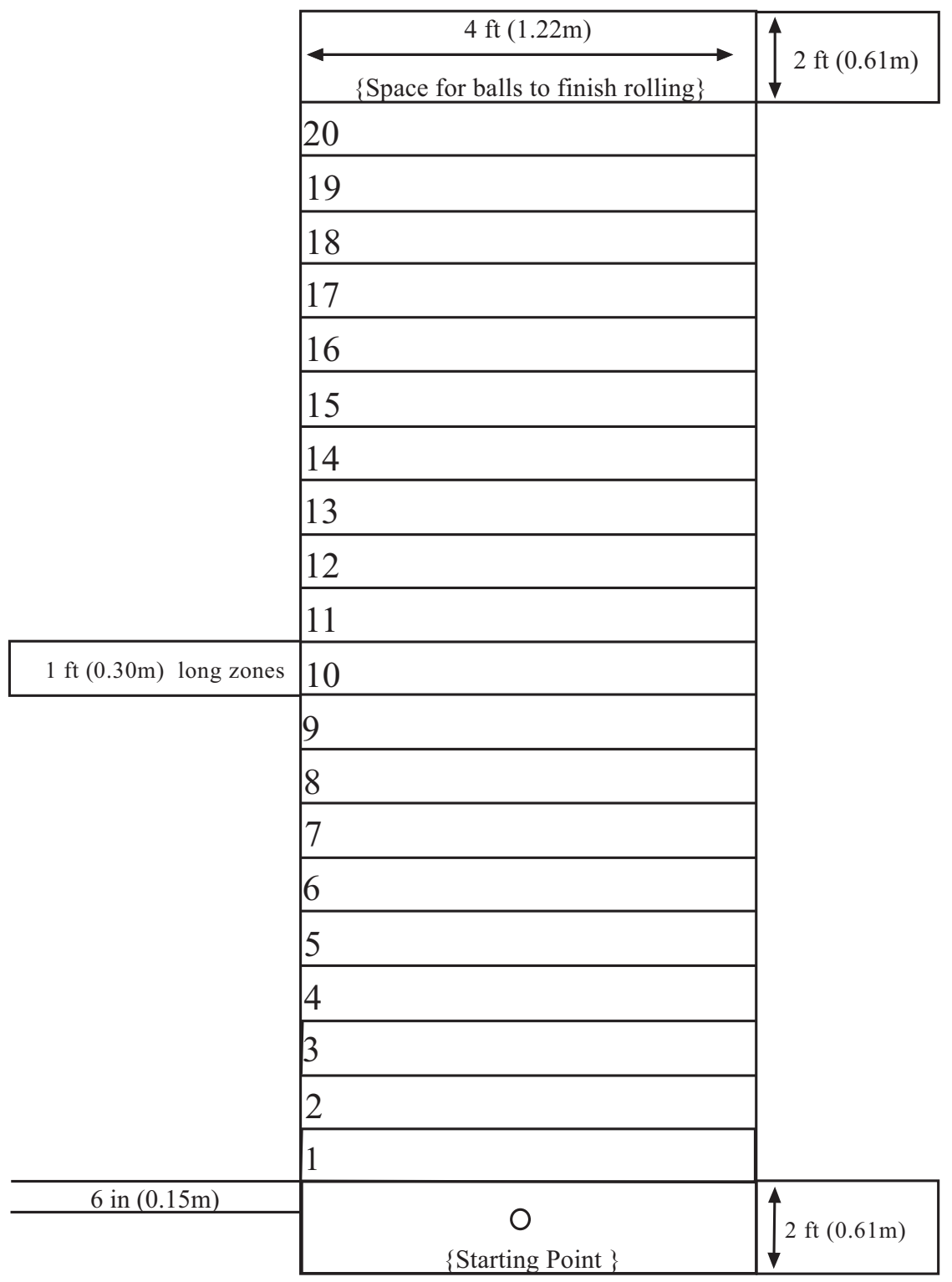

Fig. (1). Putting surface for study 1 . The figure is not drawn to scale.

identical except for their respective labels and the timing of the measurement.

Procedures. After obtaining consent and demographic information, researchers led participants through a 20-putt warm-up activity to familiarize them with the speed of the putting surface. The warm-up targets started at Zone 20, and each successive putt was aimed at the next closest zone until the last practice putt at Zone 1. The researcher allowed each putt to stop rolling before collecting the ball. This procedure provided participants opportunities to judge the quality of putts and develop a feel for the speed of the practice green.

After the warm-up session, the researcher determined participant self-efficacy for landing putts in each zone. Task self-efficacy for each zone was determined by asking participants to rate self-efficacy level for how many putts, out of a total of 5 putts, he believed he could land in the target zone in question. The researcher asked each participant, "Given a reasonable number of practice putts, how many putts out of five can you land, or properly putt to roll to a stop, in Zone 1?" Following the participant's response (i.e., a number 0 through 5), the examiner continued the procedure for each successive zone. When the participant responded that he could only pace one out of five putts to land in the given zone, the examiner asked, "Which is the farthest zone in which you can correctly pace only one out of five putts?"

Target zones were labeled in the follow manner: The farthest zone in which the participant indicated he could land five out of five putts, the high self-efficacy zone, was marked with a poker chip labeled 5. The farthest zone indicated for three out of five putts was marked with a chip labeled 3 , indicating the medium efficacy zone. The farthest zone in which the participant indicated he could only land one out of five putts was marked with a chip labeled 1, indicating the low efficacy zone. Thus, the distance of the putt varied according to the efficacy level of each 
Table 1. Experiment 1 Self-Efficacy, Effort, and Performance Data by Target

\begin{tabular}{|c|c|c|c|c|c|c|}
\hline & \multicolumn{6}{|c|}{ Targets } \\
\hline & \multicolumn{2}{|c|}{ High Efficacy } & \multicolumn{2}{|c|}{ Medium Efficacy } & \multicolumn{2}{|c|}{ Low Efficacy } \\
\hline & $M$ & $S D$ & $M$ & $S D$ & $M$ & $S D$ \\
\hline Preparatory Efficacy Level & 5 & -- & 3 & -- & 1 & -- \\
\hline Preparatory Efficacy Strength & 8.3 & 1.7 & 5.9 & 1.2 & 4.1 & 1 \\
\hline Practice Effort (putts) & 6.5 & 3 & 10.1 & 3.9 & 13.4 & 5.5 \\
\hline Performance Efficacy Strength & 8.2 & 1.5 & 5.8 & 1.4 & 3.6 & 1.2 \\
\hline Competitive Performance & 4.2 & 1.1 & 2.7 & 1.2 & 1.1 & 0.9 \\
\hline
\end{tabular}

participant. This procedure standardized efficacy levels for the experiment, which allowed efficacy level to be used as an independent variable in the study.

Next, the researcher read a scripted explanation of the practice session and competition, including an explanation of the goals of the putting competition (i.e., to earn the highest point total possible out of 15 with one point awarded for each ball landing within the intended target zone). Participants then completed the preparatory efficacy strength scale.

After completing the preparatory efficacy strength scale, participants engaged in free-choice practice putting (i.e., preparatory effort). Each participant was given 30 balls to putt during practice. The number of practice putts was determined through pilot testing. Given that each putt could only be directed at one target, participants stated the intended target for each putt. The researcher recorded each practice attempt, noting whether the ball landed in or out of the intended target zone as a measure of practice putt quality. For each participant, practice effort was defined as the number of practice putts at each target zone.

The last practice putt signaled the end of practice phase and the beginning of the performance phase. First in the performance phase, participants completed the performance efficacy strength scale. When finished with this scale, participants took five performance putts at each of the three targets, randomly presented, with all five putts being taken at a single zone before moving onto the next. The examiner recorded each performance putt as landing either in or out of the target zone, and participants earned a point for each putt that landed in the correct target zone. After the 15 performance putts, the examiner debriefed each participant and offered him an informational pamphlet on golf confidence.

\section{Results}

Manipulation check. Table 1 lists Experiment 1 data. To determine whether the targets functioned as distinct challenges, a manipulation check was performed on preparatory and performance efficacy strengths and competitive performance. Mean preparatory efficacy strengths were lowest at the low efficacy target, moderate at the medium efficacy target, and highest at the high efficacy target. Mauchly's Test of Sphericity, $W=.89, \chi 2(2)=2.69$, $p=.261$, was not significant. Results of the ANOVA,
$F(2,46)=90.77, p<.001$, were significant. Paired $t$ tests differentiated between the high and medium efficacy conditions, $t(23)=7.75, p<.001$, the high and low efficacy conditions, $t(23)=11.72, p<.001$, and the medium and low efficacy conditions, $t(23)=6.86, p<.001$.

Performance efficacy strengths were slightly lower at each target but followed the same general pattern. Significance test results were similar to those of preparatory efficacy. Mauchly's Test of Sphericity, $W=.94, \chi^{2}(2)=$ $1.38, p=.502$, was not significant. ANOVA results, $F(2,46)$ $=93.30, p<.001$, were significant. Paired $t$ tests indicated significant differences between the high and medium efficacy conditions, $t(23)=7.62, p<.001$, the high and low efficacy conditions, $t(23)=12.23, p<.001$, and the medium and low efficacy conditions, $t(23)=6.96, p<.001$.

Effort. Table 1 contains effort data in the practice phase. Repeated measures ANOVA results, $F(2,46)=10.50, p<$ .001 , confirmed significant effort differences among the targets. Mauchly's Test of Sphericity, $W=.49, \chi^{2}(2)=$ $15.52, p<.001$, was significant; however, results of Greenhouse-Geisser, $F(1.33,30.53)=10.50, p=.001$, and Huynh-Feldt, $F(1.38,31.68)=10.50, p=.001$, corrections remained highly significant. Paired $t$-tests indicated significant differences between the low and high efficacy targets $t(23)=4.24, p<.001$, and the medium and high efficacy targets $t(23)=4.28, p<.001$. The effort difference between the low and medium efficacy targets was not significant, $t(23)=1.76, p=.092$. Thus, the high efficacy condition resulted in significantly less practice effort compared to the low and medium efficacy targets.

Apart from significance testing, effect sizes [ES $=(\mathrm{M} 1-$ M2)/pooled SD] indicated large effort differences between all targets. The largest effect size was obtained between the low and high efficacy targets, ES $=1.67$, followed by the difference between the medium and high efficacy targets, ES $=0.87$, and the difference between the low and medium efficacy targets, $\mathrm{ES}=.80$.

Finally, given the linear shape of the effort increases across the conditions from the high efficacy target through the low efficacy target, a linear contrast ANOVA was employed to test the data for a linear effect for preparatory efficacy level on effort. The linear effect was significant, $F(1,69)=31.45, p<.001$, and the deviation from linear was not significant, $F(1,69)=0.31, p=.86$. Thus, effort increased in a linear trend from lowest effort at the high 
preparatory efficacy target, to moderate effort at the medium preparatory efficacy target, to highest effort at the low preparatory efficacy target.

Performance. Hypothesis 2 stated that low effort at the high efficacy target would lead to worse than expected performance (i.e., expected performance was 5 out of 5 putts in the high efficacy target zone), and higher effort at the medium and low effort targets would yield performance (i.e., 3 putts within the medium efficacy target zone and 1 putt within the low efficacy target zone) within sampling error of expectations. Performance results are listed along with expected results in Table 1. Absolute performance was best at the high efficacy target, moderate at the medium efficacy target, and worst at the low efficacy target. Mauchly's Test of Sphericity, $W=.94, \chi^{2}(2)=1.32, p=.517$, was not significant. ANOVA results, $F(2,46)=54.81, p<.001$, were significant. Paired $t$-tests indicated significant differences between the high and medium efficacy conditions, $t(23)=$ $5.09, p<.001$, the high and low efficacy conditions, $t(23)=$ $9.53, p<.001$, and the medium and low efficacy conditions, $t(23)=6.05, p<.001$. According to ANOVA results, $F(3$, 19) $=0.29, p=.83$, target order did not significantly affect performance.

In finding the difference between observed minus expected performance, the largest average difference happened at the high efficacy target, where participants underperformed by 0.8 putts $(M=-0.8, S D=1.1)$. Participants also slightly underperformed at the medium efficacy target $(M=-0.3, S D=1.2)$. Participants outperformed expectations slightly at the low efficacy target $(M=0.1, S D=0.9)$. Significant ANOVA results, $F(2,46)=$ $4.86, p=.012$, indicated differences in performance respective to expectations at the various targets. Mauchly's Test of Sphericity, $\mathrm{W}=.94, \chi^{2}(2)=1.32, p=.517$, was not significant. One-sample $t$-tests indicated that differences in observed minus expected performance were significant at only the high efficacy target, $t(23)=-3.65, p=.001$.

\section{DISCUSSION}

The main hypothesis, that the lowest effort would be directed at the high efficacy target, a moderate effort would be directed at the medium efficacy target, and the highest effort would be directed at the low efficacy target, was largely supported by the data. The least effort was directed at the high efficacy target by a significant degree, and although the effort directed at low efficacy target did not significantly surpass the effort directed at the medium efficacy target, effect size differences were large between all targets. Further, linear trend data were significant for effort increases in the proposed direction. Thus, the data were consistent with the idea that some sense of self-doubt was beneficial to practice effort, and in fact, the higher the doubt in this case, the higher the effort allocation.

Regarding performance, as hypothesized, participants underperformed in comparison to expectations at the high efficacy target only. Based on Bandura's [1] conceptualization of preparatory efficacy and the effort data, this underperformance is interpreted to be caused by a lack of adequate effort put forth in preparation for performance at this target.
In considering limitations, the interval scale scoring method had limited utility. It was helpful to use target zones to establish standardized preparatory efficacy levels, but it was problematic for performance scoring. For example, a putt that missed the target zone by $1 \mathrm{in}$. counted the same as a putt that missed by several ft. Even though the quality of those two putts was vastly different, the resulting performance score was the same. A ratio performance scale would improve the measurement of putt quality.

Further, although participants were informed that they were in a competition, little fanfare was made to create an ostensibly competitive atmosphere. The researchers considered that a simple change in incentive might be enough to create a greater semblance of competition while not changing too many other variables in the study design (e.g., emotional and social reactions to others present in the environment), such as might be the case if participants were tested together or pitted in head to head competition.

Finally, the astute critic could argue that the results of the study are consistent with goal theory [30] in that the most effort was allocated toward more difficult targets. The results are not consistent with this conjecture. Researchers created a goal for the participants in the competition: maximize total points in the competition by landing as many balls as possible in the appropriate target zones. Because each putt landing in any target zone was worth one point, regardless of whether it was the high, medium, or low efficacy zone, it would have made sense for participants to maximize competitive putts landing in the target zones at the high and medium efficacy targets, which were the easiest target zones to land in by both expected and actual performance numbers. Therefore, goal difficulty would have predicted a more uniform or even opposite distribution of effort. However, the researchers did not assess whether participants endorsed or self-set any type of goal. Therefore, to address these limitations, researchers conducted a second experiment.

\section{EXPERIMENT 2}

\section{Overview}

Given the limitations of Experiment 1, a ratio scale of performance, a lack of true competitive incentive, and a lack of self-set goal assessment, the design of Experiment 2 improved the performance measure, created a more competitive situation, and assessed self-set goals while it replicated the basic design of a putting competition utilizing three distinct targets. First, researchers recruited a larger sample, lowered the golf experience minimum to 1 year experience, and offered a $\$ 25$ prize to the top four participants in the competition in order to create a true competitive environment. Second, the task was changed from putting to a target zone to putting to a small specific target (i.e., a bullseye target). This change allowed for ratio measurement of performance, which is a more accurate indicator of performance than the interval performance measure used in Experiment 1. Also, rather than standardizing the targets according to perceived difficulty, pilot testing was employed to create three targets of distinctly different difficulties. The three targets remained the same for all participants. Fourth, the putting surface was changed to an extremely fast indoor putting green. Even 


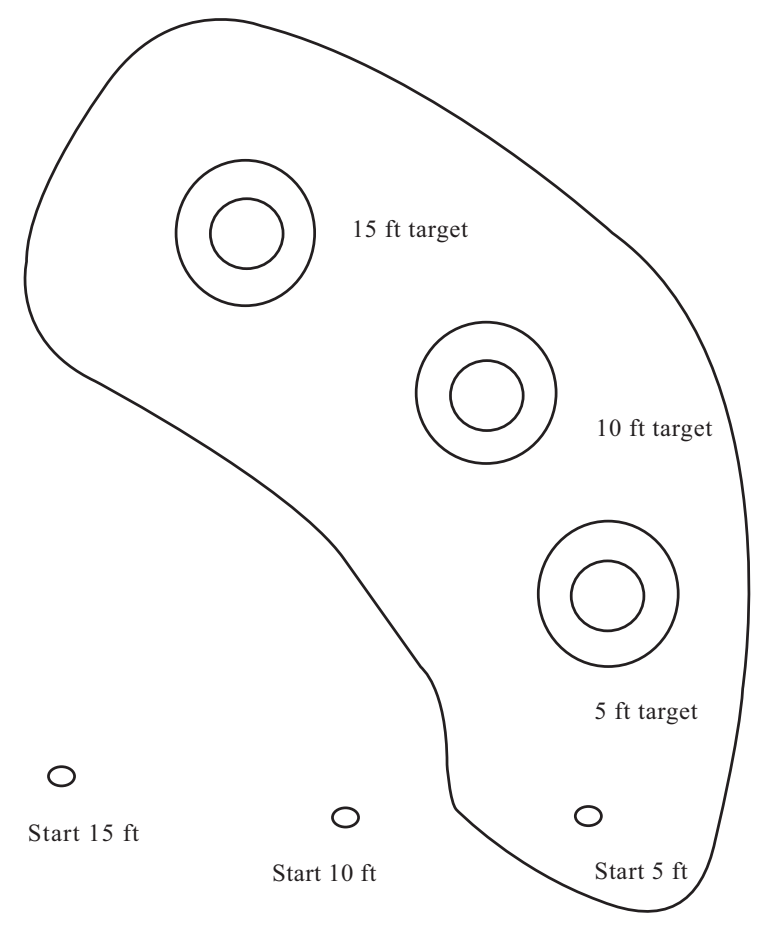

Fig. (2). Putting surface for study 2. The figure is not drawn to scale. Not all Concentric target ring are shown.

balls struck very softly tended to roll a good distance on the faster surface, and differences in putting strikes resulted in bigger distance differences on the green in comparison to the putting surface used in Experiment 1. The faster surface ensured that even the shortest putt would be challenging to land very close to the target. This change addressed the possible critique that the high efficacy target in Study 1 was simply too easy to warrant effort. Finally, self-set goals were measured to determine any possible goal effects.

The main hypothesis for Experiment 2 was identical to the hypothesis for Experiment 1. In essence, Study 2 addressed whether participants allocated differential effort to targets of differential difficulty when all targets were challenging. Performance was hypothesized to improve from baseline to performance consistent with the amount of hypothesized effort directed at each of the targets. Therefore, the largest improvement was hypothesized at the low efficacy target, a moderate improvement was hypothesized at the medium efficacy target, and the least improvement was hypothesized at the high efficacy target. Finally, self-set goals were assessed to determine whether goal effects had an impact on effort allocation or performance. Consistent with the interpretation in Experiment 1, it was hypothesized that self-set goals would not have an impact on effort.

\section{Method}

Participants. Following approval from the authorized IRB, the researchers recruited 33 male participants from a golf training program and a golf league in the Midwest. Only one year of golf experience was required for participation. Participant age averaged 35.0 years $(S D=6.4$ years), and playing experience averaged 17.4 years $(S D=6.7$ years $)$.
Age ranged from 18 to 44 years. Competitive experience levels included 14 league, 8 high school, 1 college, and 1 local professional competitor. Nine participants did not have any competitive experience. Eighteen participants provided a current handicap index $(M=-10.0, S D=7.2)$. Handicaps ranged from +2.2 to -28 (note: positive handicaps indicate higher golf skill, negative handicaps indicate lower golf skill).

Golf putting materials. The golf balls and the putter were the same as used in Experiment 1. The main putting surface was a kidney bean shaped putting green made of astroturf style carpeting with a sand filled base. The sand filled base created a putting surface very similar to a natural grass putting green with very short blades of astroturf grass rising above the sandy base. The surface was extremely fast, meaning balls rolled over it easily even when struck with minimal force. This putting surface was slightly raised (less than 1 in., $2.54 \mathrm{~cm}$ ) above the surrounding surface, which was made of regular Astroturf style carpeting, a slower speed putting surface. To increase the difficulty and length of the medium and low self-efficacy putts, the starting points to those respective putts were placed on the adjacent slower speed green. The main putting surface was marked with three bullseye targets, each made of a 1 in. $(2.54 \mathrm{~cm})$ circular shaped sticker adhered to the putting surface. The respective targets were spaced at $5 \mathrm{ft}(1.52 \mathrm{~m}), 10 \mathrm{ft}(3.05 \mathrm{~m})$, and $15 \mathrm{ft}$ $(4.57 \mathrm{~m})$ from the starting point. Four concentric circles surrounded each target and provided visual feedback on putt quality. The outer target circles were $1 \mathrm{ft}(0.30 \mathrm{~m}), 2 \mathrm{ft}(0.61$ $\mathrm{m}), 3 \mathrm{ft}(0.91 \mathrm{~m})$, and $4 \mathrm{ft}(1.22 \mathrm{~m})$ in diameter. Thus, the outer circles for each target indicated points 6 in. $(0.18 \mathrm{~m}), 1$ $\mathrm{ft}(0.37 \mathrm{~m}), 1 \mathrm{ft} 6$ in. $(0.46 \mathrm{~m})$, and $2 \mathrm{ft}(0.61 \mathrm{~m})$ from the centered bullseye. All distances in the study were measured with a triangular meter stick (i.e., a hollow triangle $1.00 \mathrm{~m}$ long). Because the meter stick was marked at the $\mathrm{m}$, $\mathrm{cm}$, and $\mathrm{mm}$ level on all sides and because it touched the midpoint of the ball in a consistent way when laid flat on the ground, the triangle shape was excellent for measuring distances on the ground and distances from a target on the ground to the midpoint of the ball. The putting surface for Study 2 is illustrated in Fig. (2).

Preparatory and performance efficacy strength scales. Only time of measurement differentiated the preparatory and performance efficacy scales. The scales required self-efficacy ratings regarding each participant's ability to land putts within a specified distance of the target. Participants rated efficacy strength for being able to obtain an average putt length within $2 \mathrm{ft}, 1 \mathrm{ft} 6$ in., $1 \mathrm{ft}$, and 6 in. of each target. Responses to the four items were averaged to obtain an efficacy strength score for each target. The selfefficacy scale anchors were labeled Complete Uncertainty, Moderate Certainty, and Complete Certainty and respectively centered above 0,5 , and 10 on the scales.

Goals. Self-set goals were measured via a questionnaire. Participants described self-set goals in an open ended format. Goal data were coded according to type of self-set goal indicated.

Procedures. The researcher informed participants of the putting contest in which the top four performers would receive \$25 dollar gift certificates to a local golf store. After 
Table 2. Efficacy, Performance, and Effort Data by Target

\begin{tabular}{|c|c|c|c|c|c|c|}
\hline & \multicolumn{6}{|c|}{ Targets } \\
\hline & \multicolumn{2}{|c|}{ High Efficacy } & \multicolumn{2}{|c|}{ Medium Efficacy } & \multicolumn{2}{|c|}{ Low Efficacy } \\
\hline & $M$ & $S D$ & $M$ & $S D$ & $M$ & $S D$ \\
\hline Preparatory Efficacy & 7.4 & 1.4 & 5.6 & 1.7 & 4.3 & 1.7 \\
\hline Performance Efficacy & 7.3 & 1.7 & 4.8 & 1.7 & 3.8 & 2 \\
\hline Baseline Performance & 25.9 & 8.8 & 59.7 & 18.4 & 73.8 & 25.5 \\
\hline Competitive Performance & 20.2 & 7.3 & 49.2 & 19.5 & 66 & 22.5 \\
\hline Practice Effort & 7.4 & 2.5 & 10.9 & 2.7 & 11.7 & 3.5 \\
\hline
\end{tabular}

obtaining consent, demographic information, self-set goals, and baseline putting self-efficacy measures, researchers explained the objective of the putting contest: putt the ball to stop it as close as possible to the bullseye target. It was noted that good performances consisted of landing putts near the targets and avoiding putts $100 \mathrm{~cm}$ or farther from the targets.

To begin, researchers obtained baseline putting performance measures. Participants putted five balls at each target, starting with 5 putts at the high self-efficacy target before moving onto the medium self-efficacy target and then the low self-efficacy target. The researcher recorded putt lengths from target to the nearest tenth of a centimeter and also announced the lengths aloud to provide putt performance feedback to the participants. Additionally, the outer target circles provided visual feedback regarding putt performance.

After the baseline putts, participants completed the preparatory efficacy scales. Participants then took 30 free choice putts at the three targets similar to the procedure in Study 1. However, unlike in Study 1, the researcher measured the distance of each practice putt from the target to obtain a measure of practice putt quality, and participants privately recorded a putt satisfaction rating. As in Study 1, the researcher recorded the number of practice putts at each target as a measure of practice effort.

At the conclusion of the 30 practice putts, the researcher announced that the practice phase had ended and the competitive performance phase had begun. Participants then filled out the performance efficacy and post-practice satisfaction scales. After completing the scales, participants putted five balls at each target as a measure of competitive performance. The order and measurement procedures used for the competitive putts were identical to the baseline procedures. Finally, participants completed the performance satisfaction scale before being debriefed.

\section{Results}

Manipulation check. Table 2 lists Experiment 2 data. Self-efficacy data substantiated three targets of distinct difficulty. ANOVA results indicated significant differences among preparatory efficacy strengths, $F(2,64)$, $=150.50$, $p<.001$, performance efficacy strengths, $F(2,64),=104.89$, $p<.001$, Mauchly's Test of Sphericity revealed violations for each of these variables; however, Greenhouse-Geisser and Huynh-Feldt corrections revealed unchanged $p$ values.
Post-hoc $t$-tests indicated specific differences between the targets. Regarding preparatory efficacy, $t$-tests indicated that preparatory efficacy was significantly higher at the high target than at the low target, $t(32)=14.12, p<.001$, significantly higher at the medium compared to the low efficacy targets, $t(32)=7.87, p<.001$, and significantly higher at the high efficacy compared to medium efficacy target, $t(32)=12.06, p<.001 . T$-tests also indicated that performance efficacy at the high target was significantly higher than at the low efficacy targets, $t(32)=11.56$, $p<.001$, significantly higher at the medium compared to the low efficacy targets, $t(32)=5.01, p<.001$, and significantly higher at the high compared to the medium efficacy target, $t(32)=10.50, p<.001$.

Effort. Repeated measures ANOVA results revealed significant effort differences between the three targets, $F(2)$ $=13.32{ }_{2} p<.001$. Although Mauchly's Test of Sphericity, $W$ $=.82, \chi(2)=6.17, p=.046$, was significant, GreenhouseGeisser, $F(1.69,54.22)=13.32, p<.001$, and Huynh-Feldt, $F(1.78,56.93)=13.32, p<.001$, corrections remained significant at the same level. As hypothesized, effort at the high efficacy target was significantly lower than effort at the medium, $t(32)=-5.13, p<.001$, and low efficacy targets, $t(32)=-4.58, p<.001$. However, effort at the medium and low efficacy targets was not significantly different, $t(32)=$ $-84, p=.406$, replicating the results from Experiment 1 .

Using the effect size calculation from Experiment 1 [ES $=(\mathrm{M} 1-\mathrm{M} 2) /$ pooled SD], the largest effort difference occurred between the low and high efficacy targets, ES = 1.5 , and the second largest difference occurred between the medium and high efficacy targets, $\mathrm{ES}=1.2$. Both were large effects. The effort difference between the low and medium efficacy targets resulted in a low effect size, $\mathrm{ES}=0.3$.

As with Study 1, a linear effect of preparatory efficacy on effort was tested. The linear effect was significant, $F(1,69)$ $=16.03, p<.001$, while the deviation from linear was not, $F(28,69)=1.24, p=.24$.

Performance. Similar to the self-efficacy results, ANOVA results substantiated three distinct targets of varying difficulty for both baseline performance, $F(2,64)$, = $50.28, p<.001$, and competitive performance, $F(2,64)$, = $63.99, p<.001$. Regarding baseline putting performances, $t$-tests indicated significant differences between baseline putting performances at the high and low efficacy targets, $t(32)=-9.43, p<.001$, the medium and low efficacy targets, 
$t(32)=-2.43, p=.021$, and the high and medium efficacy targets, $t(32)=-9.39, p<.001$. Likewise, competitive performances differed significantly at the high and low efficacy targets, $t(32)=-10.96, p<.001$, the medium and low efficacy targets, $t(32)=-3.81, p=.001$, and the high and medium efficacy targets, $t(32)=-7.93, p<.001$. From the preparatory stage to competitive performance, putting performance improved significantly at the high efficacy target, $t(32)=-2.75, p=.010$, and medium efficacy target, $t(32)=-2.22, p=.033$, but not at the low efficacy target, $t(32)=-1.51, p=.142$. Thus, the performance hypothesis was partially supported.

Goals. Goal data were coded according to type. Ten participants set no goals. Seven set goals to improve from baseline to performance. Six participants set do your best type goals for maintaining equally good performance across all three targets. An example of this type of goal would be to average performance putts within $1 \mathrm{ft}$. of each target. Five participants set goals for increasing the accuracy of putts as the target distance shortened. In other words, a typical goal of this type was to average performance within 6 in. of the high efficacy target, $1 \mathrm{ft}$. or less of the medium efficacy target, and $1.5 \mathrm{ft}$. of the low efficacy target. Four participants set outcome type goals for winning or placing in the final competition. One participant set a process type for a good tempo and follow through stroke. None of the participants self-set multiple types of goals. Goal data did not support the idea that a high percentage of participants were practicing according to any specific goal based strategy.

\section{DISCUSSION}

In Experiment 2, a significant linear increase in effort was observed across the high, medium, and low efficacy targets. Effort at the medium and low efficacy targets were both significantly greater than effort at the high efficacy target and had large effect sizes when compared with the high efficacy target. Thus, as with Experiment 1, the data are consistent with the idea that a higher sense of doubt resulted in greater practice effort. However, the effort difference between the low and medium efficacy targets was not significantly different, and obtained only a small effect size of 0.3. Thus, higher levels of preparatory doubt did not necessarily lead to greater practice effort. Reasons for this lack of significance are offered in the General Discussion section.

Performance data indicate that the smallest improvement from baseline to performance indeed occurred at the target that participants spent the least effort practicing, the high efficacy target. However, contrary to the performance hypothesis, the most improvement occurred at the medium efficacy target, and a moderate improvement occurred at the low efficacy target. Thus, performance data did not completely conform to the idea that more practice effort equals better performance.

In considering the performance data, it may be helpful to reconsider Bandura's [1] initial conception of preparatory efficacy and Feltz and Wood's [23] interpretation that high practice effort is desired because it should lead to high quality practice behaviors, which serve as mastery experiences, the most important source of self-efficacy beliefs $[1,2]$. Thus, practice effort is important when investigating preparatory efficacy, but practice quality must be considered as well.

In preliminary support of this idea, efficacy changes from preparation to performance were highly related to practice quality $(r=-.47, p=.005)$. Eleven participants reported increased efficacy strength following the practice putting session. On average, the performance of the 11 participants who increased efficacy from preparation to performance ( $M$ $=39.8, S D=9.8)$ was better than the performance of the 21 participants who decreased efficacy from preparation to performance $(M=47.7, S D=11.7)$ by an effect size of 0.73 . Thus, practice quality appears to be an important feature of the preparatory efficacy concept. Future studies utilizing manipulated practice quality are recommended to study this phenomenon and further explore preparation efficacy performance relationships, especially in regard to whether the efficacy and performance changes are accompanied by actual changes in skill. Given the considerable experience of the participants in this study and the difficulty of changing actual putting skill, skill level is assumed not to have changed significantly within the 60 putts taken in this study; however, this is an empirical question for future study.

Participants did not set consistent goals that would affect the outcome of the experiment in a specific way. Coding techniques categorized the self-set goals into six different categories. The most frequently used goals included none, do your best, and improve competitive putts from baseline. Thus, the results of the goal data do not support one seemingly valid criticism of the findings. The data are not explicable through goal theory.

\section{GENERAL DISCUSSION}

Data from the two experiments support Bandura's [1] prediction that some sense of doubt in preparatory efficacy is beneficial to effortful practice in preparation for competition. In both experiments, the lowest effort occurred at the high efficacy target, medium effort occurred at the medium efficacy target, and the highest effort occurred at the low efficacy target. Effort increased across the targets in a significant linear trend in both experiments. In Experiment 1, large ES differences occurred between all targets, and in Experiment 2, large ES differences were obtained between the high and medium efficacy targets and the high and low efficacy targets.

Despite the linear effort increases across decreasing preparatory efficacy conditions in both experiments, practice effort differences between the medium and low efficacy targets were not significant in either experiment. While not significant, the effort increase from the medium to the low efficacy target obtained a large ES in Experiment 1 $(\mathrm{ES}=0.8)$; however, the effort increase was smaller in Experiment $2 \quad(\mathrm{ES}=0.3)$. Although not hypothesized in Experiment 2, the small effort difference between the medium and low efficacy targets is consistent with Feltz and Wood's [23] supposition that preparatory efficacy-effort relationship takes the general form of an inverted-U. This reasoning is based on the idea that some possible preparatory activities are either too easy or too difficult to warrant effort 
expenditure. If the relationship between preparatory efficacy beliefs and effort is indeed an inverted-U, effort must peak at some point, presumably in the moderate to low preparatory efficacy range. The small effort difference between the medium and low efficacy targets in Experiment 2 may be a manifestation of this peak.

Experiment 1 left open the possibility that participants were simply allocating more effort toward more difficult goals, which would be the result hypothesized by goal theories. This explanation did not fit with the stated goals of the task in Experiment 1, but without direct measurement of goals in Experiment 1, researchers decided to survey participants about their goals in Experiment 2. When examined, no evidence from Experiment 2 indicated that participants were simply allocating more effort toward more difficult goal conditions. The evidence strongly favored the idea that participants allocated more effort toward the medium and low efficacy targets, and the goal data indicated that participants had not set more difficult goals for these targets.

Future studies should include longer time frames of preparation-performance cycles, larger samples of athletes across various sports and task difficulty levels, inclusions of skill measures when actual skill is likely to change, and various effort and persistence methods and measurements. Further, the experiments described in this article only investigated preparatory efficacy related to task difficulty. Athletes in competitive sports not only deal with varying task difficulty, they deal with varying opponent difficulty. Thus, future studies should examine preparatory competitive efficacy as well as preparatory task efficacy. Finally, as observed in Experiment 2, practice quality is an important variable in preparatory efficacy research and should be included in future preparatory efficacy studies.

In conclusion, doubt is a natural component of sport performance in which information that has the potential to decrease self-efficacy beliefs is readily available, especially to the over $50 \%$ of individual and team sport athletes who lose or finish lower than first place in any given competition. Accurate assessment of strengths and weaknesses, which may include an assessment of self-doubt regarding capabilities, is essential to competition strategy and skill development. The results of the current experiments suggest that doubt may play a motivating role in allocating effort toward practicing to improve aspects of performance for which self-efficacy is relatively low.

Despite the potential benefits of doubt, caution is warranted when applying these findings to athlete preparation in the field. Because doubt has not been supported adequately for performance in sport it may be wise to remember that doubt can be effectively reframed as challenge. When an athlete has doubt, it is because a challenge to personal capability is present. The point at which a great challenge creates overwhelming doubt is perhaps a question for future research, but the coach or sport psychology consultant applying the principles of preparatory efficacy would be wise to remember the sentiment of Bandura and Cervone [25] quoted in the Introduction to this article. Preparatory doubt may serve as a signal that a worthy challenge is present and spur athletes to strong preparatory effort; however, doubt tends not to be beneficial to performance [22]. As was observed in Experiment 2, an efficacious mindset, especially one supported by mastery experiences, tends to be best for competition.

The research contained herein suggests that providing challenging but manageable practice tasks is perhaps one safe way to introduce doubt and increase effortful practice behaviors that lead to mastery experiences for the athlete. In turn, mastery experiences should improve true skill, provide opportunities for athletes to become aware of self-efficacy increasing feedback (e.g., mastery, modeling, verbal persuasion from coaches or teammates), and create opportunities to gather information on strengths and weaknesses, which may prove fruitful to creating effective game plans and strategies. Thus, the balance between challenge, preparatory efficacy, and mastery experiences is extremely important for choosing practice activities that provide ample opportunity for mastery experiences while creating enough challenge to overcome the detrimental preparatory effort decreases commonly attributed to overconfidence.

\section{CONFLICT OF INTEREST}

The authors confirm that this article content has no conflicts of interest.

\section{ACKNOWLEDGEMENT}

Declared none.

\section{REFERENCES}

[1] Bandura A. Self-efficacy: The exercise of control. New York: W.H. Freeman and Company 1997.

[2] Feltz DL, Short SE, Sullivan PJ. Self-efficacy in Sport: Research and strategies for working with athletes, teams, and coaches. champaign, IL: Human Kinetics 2008.

[3] Feltz DL. A path analysis of the causal elements in Bandura's theory of self-efficacy and an anxiety-based model of avoidance behavior. J Pers Soc Psychol 1982; 42: 764-81.

[4] Feltz DL, Lirgg CD. Perceived team and player efficacy in hockey. J Appl Psychol 1998; 83: 557-64.

[5] Feltz DL, Mugno DA. A replication of the path analysis of the causal elements in Bandura's theory of self-efficacy and the influence of autonomic perception. J Sport Psychol 1983; 12: 13243.

[6] George TR. Self-confidence and baseball performance: A causal examination of self-efficacy theory. J Sport Exerc Psychol 1994; 16: 381-99.

[7] McAuley E. Modeling and self-efficacy: A test of Bandura's model. J Sport Psychol 1985; 7: 283-95.

[8] Myers ND, Feltz DL, Short SE. Collective efficacy and team performance: A longitudinal study of collegiate football teams. Group Dyn 2004; 8: 126-38.

[9] Myers ND, Payment CA, Feltz DL. Reciprocal relationships between collective efficacy and team performance in women's ice hockey. Group Dyn 2004; 8: 182-95.

[10] Weinberg RS, Gould D, Jackson A. Expectations and performance: An empirical test of Bandura's self-efficacy theory. J Sport Psychol 1979; 1:320-31.

[11] Weinberg RS, Gould D, Yukelson D, Jackson A. The effects of pre-existing and manipulated self-efficacy on a competitive muscular endurance task. J Sport Psychol 1981; 3: 345-54.

[12] Locke EA, Frederick E, Lee C, Bobko P. Effect of self-efficacy, goals, and task strategies on task performance. J Appl Psychol 1984; 69: 241-51.

[13] Kane TD, Marks MA, Zaccaro SJ, Blair V. Self-efficacy, personal goals, and wrestler's self-regulation. J Sport Exerc Psychol 1996; 18: 36-48. 
[14] Hepler TJ, Feltz DL. Path analysis examining self-efficacy and decision making performance on a simulated baseball task. Res Q Exerc Sport 2012; 83: 55-64.

[15] Treasure DC, Monson J, Lox CL. Relationship between selfefficacy, wrestling performance, and affect prior to competition. Sport Psychol 1996; 10: 73-83.

[16] Feltz DL, Chow GM, Hepler TJ. Path analysis of self-efficacy and diving performance: Revisited. J Sport Exerc Psychol 2006; 28: S66.

[17] Vancouver JB, Thompson CM, Williams AA. The changing signs in the relationships between self-efficacy, personal goals, and performance. J Appl Psychol 2001; 86: 605-20.

[18] Vancouver JB, Thompson CM, Tischner EC, Putka DJ. Two studies examining the negative effect of self-efficacy on performance. J Appl Psychol 2002; 87: 506-16.

[19] Woodman T, Akehurst S, Hardy L, Beattie S. Self-confidence and performance: A little self-doubt helps. Psychol Sport Exerc 2010; 11: 467-70.

[20] Beattie S, Lief D, Adamoulas M, Oliver E. Investigating the possible negative effects of self-efficacy upon golf putting performance. Psychol Sport Exerc 2011; 12: 434-41.

[21] Weinberg RS, Gould D. Foundations of sport and exercise psychology. $4^{\text {th }}$ ed. Champaign: Human Kinetics 2007.

[22] Moritz SE, Feltz DL, Fahrbach KR, Mack DE. The relation of selfefficacy measures to sport performance: A meta-analytic review. Res Q Exerc Sport 2000; 71: 280-94.
[23] Feltz DL, Wood JM. Can self-doubt be beneficial to performance? Exploring the concept of preparatory efficacy. Open Sports Sci J 2009; 2: 65-70.

[24] Bandura A, Cervone D. Self-evaluative and self-efficacy mechanisms governing the motivational effects of goal systems. J Pers Soc Psychol 1983; 45: 1017-28.

[25] Bandura A, Cervone D. Differential engagement of self-reactive influences in cognitive motivation. Organ Behav Hum Dec Process 1986; 38: 92-113.

[26] Bandura A, Jourdan FJ. Self-regulatory mechanisms governing the impact of social comparison on complex decision making. J Pers Soc Psychol 1991; 60: 941-51.

[27] Salomon G. Television is easy and print is tough: The differential investment of mental effort in learning as a function of perceptions and attributions. J Educ Psychol 1984; 76: 647-58.

[28] Stone D. Overconfidence in initial self-efficacy judgments: Effects on decision processes and performance. Organ Behav Hum Dec Process 1994; 59: 452-74.

[29] Eyal N, Bar-Eli M, Tenenbaum G, Pie J S. Manipulated outcome expectations and competitive performance in motor tasks with gradually increasing difficulty. Sport Psychol 1995; 9: 188-200.

[30] Locke EA, Latham GP. Building a practically useful theory of goal setting and task motivation. Am Psychol 2002; 57: 705-17.

Received: February 21, 2013

Revised: April 26, 2013

Accepted: May 29, 2013

(C) Deborah L. Feltz; Licensee Bentham Open.

This is an open access article licensed under the terms of the Creative Commons Attribution Non-Commercial License (http://creativecommons.org/licenses/ by-nc/3.0/) which permits unrestricted, non-commercial use, distribution and reproduction in any medium, provided the work is properly cited. 